Pacific

Journal of

Mathematics

SUFFICIENT CONDITIONS FOR CAPILLARY SURFACES TO BE ENERGY MINIMA

Thomas I. Vogel

Volume $194 \quad$ No. 2

June 2000 


\title{
SUFFICIENT CONDITIONS FOR CAPILLARY SURFACES TO BE ENERGY MINIMA
}

\author{
Thomas I. Vogel
}

\begin{abstract}
It is shown that if a capillary surface satisfies conditions relating to the eigenvalues of a certain differential operator, then the surface is a constrained strict local minimum for the relevant energy functional. The space of perturbations of the surface is first defined in terms of graphs of functions in curvilinear coordinates and then related to perturbations of capillary surfaces which are uniformly small and have uniformly small derivatives.
\end{abstract}

\section{Introduction.}

If a drop of liquid is put into contact with some fixed solid region $G$ with boundary $\Lambda$ and allowed to reach an equilibrium, the surface of the resulting drop is an example of a capillary surface. (A standard introduction to the study of capillary surfaces is [5].) Let $\Omega$ be the region in space occupied by the drop and $\Sigma$ be the free surface of $\Omega$. In the absence of gravity or any other external potential, the shape of the drop results from minimizing the energy functional

$$
\mathcal{E}(\Omega)=|\Sigma|-c\left|\Sigma_{1}\right|
$$

where $|\Sigma|$ is the area of the free surface, $\left|\Sigma_{1}\right|$ is the area of the wetted region on $\Lambda$ (i.e., $\partial \Omega \cap \Lambda$ ), and $c \in[-1,1]$ is a physical constant depending on the materials involved. The minimization is under the constraint that the volume is fixed. The first order necessary conditions for a drop to minimize the energy in (1.1) are that the mean curvature of $\Sigma$ is a constant $H$ and the angle between the normals to $\Sigma$ and $\Lambda$ is constantly $\gamma=\arccos (c)$ (see [5]). In this paper, we will call a surface which satisfies these conditions a capillary surface. It should be noted, however, that this is the special case of zero gravity. Another point to note is that we are not restricting ourselves to the commonly studied special case of $\Sigma$ being a graph (the non-parametric case). In view of uniqueness results (see [5], [15]), stability questions in the non-parametric case are unlikely to be interesting.

Since capillary surfaces arise from the minimization of an energy functional, it's natural to wonder whether a given configuration which satisfies the first order necessary conditions is actually a constrained minimum for the 
energy functional in some sense. In unconstrained problems, the question of whether appropriate positivity properties of the second variation yield sufficient conditions for a local minimum is well studied. For isoperimetric problems, such as the one considered in this paper, less is known. This paper is one of a series of papers $([\mathbf{1 7}],[\mathbf{1 8}],[\mathbf{1 9}])$ which use spectral methods to give sufficient conditions for a stationary point in an isoperimetric problem to be a strong local minimum. The genesis of this line of research was [6], in which Finn pointed out that the second order conditions used in $[\mathbf{1 4}],[\mathbf{1 6}],[\mathbf{2 1}]$ were insufficient to show that a given liquid bridge was an energy minimum, in spite of my erroneous assertion in [14] to the contrary.

A distinction must be made between two concepts: stability of a capillary surface and a capillary surface being a constrained strict local minimum for energy. (The latter concept was called "non-linear stability" in [19].) Stability has typically been defined as the second variation being either positive or non-negative for all volume-preserving perturbations (e.g., [2], [8], [11]). The relationship between stability and local minimality is more tenuous than one might expect from the terminology. A necessary condition for a surface to be a local minimum is that the second variation be non-negative for all volume preserving perturbations (assuming enough smoothness). However, the condition that the second variation be positive is not a priori sufficient to imply that the surface is an energy minimum. This is a standard fact from the calculus of variations, and certainly is not restricted to capillary surfaces. It does not depend on the existence of a constraint: see [1], p. 157, for an example of a function defined on $l_{2}$ whose second differential is positive definite at a critical point, but which does not have a local minimum there. Finn gives an example with similar properties in [6].

Stability and local minimality are not completely unrelated, of course. In $[8]$ it is shown that a strictly stable constant mean curvature surface is a local energy minimizer, assuming that the boundary is fixed. Strict stability, as defined in [8], is related to the idea of strong positivity in Section 2. The methods used in [8] differ from those in the present paper, and the assumption that we will make that the contact curve is free to move adds significant complications.

The result of Theorem 2.2 is that certain eigenvalue conditions are in fact enough to imply strong positivity. The eigenvalue conditions are similar to conditions which have been used for quite a while to imply that the second variation is positive (see, e.g., [10]), and which were shown in [17] to imply strict constrained local minimality. We cannot apply the results of $[\mathbf{1 7}]$ directly, however, since surface area is not a differentiable operator in the natural Hilbert space. A more delicate argument is required: it is necessary to deal with two spaces, both $H^{1}(\Sigma)$ and $C^{1}(\Sigma)$. Spectral theory in $H^{1}(\Sigma)$ will be used in Section 2 to derive sufficient conditions for a given capillary surface to be a constrained strict local minimum in $C^{1}(\Sigma)$. 
In Theorem 3.2, possibly the most important result of the paper, we will derive sufficient conditions for a capillary surface to be a strict local minimum for (1.1), subject to the volume constraint. Of course, to make such a statement, we will have to make precise the set of allowable surfaces and the sense in which two surfaces are close. Essentially, we consider perturbations of the original surface which are obtained by adding a small vector-valued function to the original parameterization of the surface, and also requiring the derivatives of this vector-valued function to be small. Of course, this means that the nearby surfaces that we compare with are fairly smooth, if the original surface is smooth. It's natural to make this assumption, since local minimizers must be smooth, assuming that the fixed solid is smooth. More precisely, interior regularity of a capillary surface must be the same as that of a minimal surface (from a blowing-up argument), so that a capillary surface in $\mathbb{R}^{3}$ will be analytic (see $[\mathbf{1 3}]$ ). Boundary regularity is also addressed by Taylor in [13]. If $\Lambda$ is at least Hölder continuously differentiable, Taylor shows that $\partial \Sigma$ consists of a finite number of curves which are also Hölder continuously differentiable. If $\Lambda$ is $C^{\infty}$, higher regularity of the contact curve $\partial \Sigma$ follows by the routine argument of straightening $\Lambda$ with a $C^{\infty}$ diffeomorphism and using elliptic theory (see, e.g., [7]).

Before getting to this point, we introduce a generalization of normal variations of surfaces. If $\vec{x}(u, v)$ is a surface with normal $\vec{N}(u, v)$, and if $\varphi(u, v)$ is a scalar function, then a new surface may be obtained by moving in the normal direction a distance $\varphi$, resulting in the surface $\vec{x}+\varphi \vec{N}$. This classic idea must be altered in dealing with capillary surfaces, since a normal variation of a capillary surface will generally not result in a physically possible surface. The problem is that the boundary of the perturbed surface need not lie on the fixed surface $\Lambda$. In Section 2 we will use curvilinear coordinates to overcome this difficulty. For a different approach to generalizing normal variations, see Section 1.5 of [5].

\section{Energy minimality in curvilinear coordinates.}

In [19], normal variations were generalized as follows. We set up a specific curvilinear coordinate system $\vec{x}(u, v, w)$ with the property that $\vec{x}(u, v, 0)$ was the unperturbed surface $\Sigma$ (which in [19] was a cylinder), and if $\vec{x}(u, v, 0)$ was on the boundary of $\Sigma$, then $\vec{x}(u, v, w) \in \Lambda$ for all $w$ sufficiently small. Then, for any sufficiently small continuous function $\varphi(u, v), \vec{x}(u, v, \varphi(u, v))$ represents the surface of a physically possible drop.

The approach we will follow will be similar to the one used in [19], however we will not restrict ourselves to a specific curvilinear coordinate system. We are given a fixed set $G$ in $\mathbb{R}^{3}$, with the boundary of $G$ being a surface $\Lambda$. We will assume that $\Lambda$ is smooth, although not necessarily connected. (It seems clear that the smoothness assumption can be weakened to consider capillary 
surfaces in wedges, although we will not do so in this paper. See [5] for discussion of several questions involving capillary surfaces in wedges.) The set $G$ corresponds to a fixed solid, to which a mass of liquid will attach. We seek to characterize surfaces $\Sigma$ surrounding regions $\Omega$ contained in the complement of $G$ so that the functional $\mathcal{E}$ of (1.1) has $\Omega$ as a strict local minimum under perturbations which preserve the volume of $\Omega$.

The general idea is the following. We set up a curvilinear coordinate system in a subset of $\left(G^{0}\right)^{\prime}$ which contains $\Sigma$. Here $G^{0}$ is the interior of $G$, and the prime denotes the complement of that set. This coordinate system $\vec{x}: \bar{\Sigma} \times[-\epsilon, \epsilon] \rightarrow\left(G^{0}\right)^{\prime}$ will be such that if $p \in \Sigma$ then $\vec{x}(p, 0)=p$ and if $p \in \partial \Sigma, \vec{x}(p, w) \in \Lambda$. We will assume that the parameterization of $\Sigma$ and the curvilinear coordinate system is such that for $(u, v)$ in a coordinate patch, $\vec{x}(p(u, v), w)$ is a smooth function of $u, v$, and $w$ which is a locally invertible map from $\mathbb{R}^{3}$ to $\mathbb{R}^{3}$. We also assume that the curvilinear coordinate system does not break down at the boundary of $\Sigma$, i.e., that $\vec{x}_{w}$ is not orthogonal to the normal to $\Sigma$ in the limit as we approach $\partial \Sigma$. (The existence of such a coordinate system is discussed in Section 3.) For any $\varphi \in C^{1}(\bar{\Sigma})$ with $|\varphi|$ bounded uniformly by $\epsilon,\{\vec{x}(p, \varphi(p)): p \in \Sigma\}$ describes an embedded surface in the exterior of $G$. Moreover, the boundary of this surface is automatically in $\Lambda$, so that this is the free surface of a physically realizable drop. It is important to note that the above assumptions on the curvilinear coordinate system exclude consideration of the cases of contact angle 0 or $\pi$.

To illustrate, here is a two dimensional example. Suppose that $G$ is $\left\{(x, y): y<x^{2}\right\}$, and $\Sigma$ is an arc of a circle which begins and ends on the parabola $\Lambda=\left\{(x, y): y=x^{2}\right\}$. Let $\vec{x}(p, w)$ be a curvilinear coordinate system as discussed above. In Figure 1 we illustrate such a coordinate system by giving curves along which the coordinates are constant. The curve $w=0$ is simply $\Sigma$, and we have chosen the coordinate system so that the curves along which $w$ is constant are also circles. As an example, we take $\varphi(p)$, $p \in \Sigma$, to be a multiple of half of a cycle of cosine. The perturbation of $\Sigma$ given by $\vec{x}(p, \varphi(p))$ will look like $\Sigma_{1}$ in Figure 1 .

Returning to the general theory, to simplify some formulas, we will also impose the condition that

$$
\vec{x}_{w}(p, 0) \cdot \vec{N}=1
$$

for all $p \in \Sigma$. Indeed, if $\vec{y}(p, w)$ is a curvilinear coordinate system as described above, we may rescale the $w$ coordinate:

$$
\vec{x}(p, w)=\vec{y}\left(p, \frac{1}{\vec{y}_{w}(p, 0) \cdot \vec{N}} w\right)
$$


to obtain the additional condition that $\vec{x}_{w}(p, 0) \cdot \vec{N}=1$. (Local invertibility will imply that $\vec{y}_{w}(p, 0) \cdot \vec{N} \neq 0$.) This will enable us to drop otherwise annoying factors of $\vec{x}_{w} \cdot \vec{N}$.

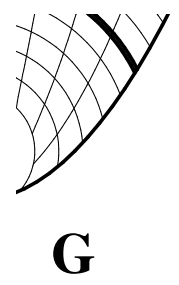

Figure 1. Example of curvilinear coordinates.

We thus have a convenient way of defining surface area and volume of drops corresponding to functions in $C^{1}(\bar{\Sigma})$ which are uniformly less than $\epsilon$. Specifically, for $\varphi \in C^{1}(\bar{\Sigma}), A(\varphi)$ will be the area of the free surface $\vec{x}(p, \varphi(p)), V(\varphi)$ will be the volume contained by the drop with free surface $\vec{x}(p, \varphi(p))$ and $W(\varphi)$ will be the area of the region on $\Lambda$ wetted by this drop. Thus, the energy corresponding to a given $\varphi$ is

$$
\mathcal{E}(\varphi)=A(\varphi)-c W(\varphi) .
$$

Since a function $\varphi$ corresponds to a perturbation of $\Sigma, \Sigma$ will have strictly less energy than nearby surfaces which are smooth graphs in curvilinear coordinates if the function which is identically 0 is a strict local minimum of (2.1). To avoid confusion with the number zero, we will label the function which is identically zero as $o$. Clearly, not all nearby surfaces can be obtained using a curvilinear coordinate system in the above fashion, if the coordinate system is fixed. However, I believe that the set of surfaces considered is large enough to be of interest. This point is discussed further in Section 3.

To use the general approach of [17] and [18], we must deal with Fréchet derivatives (in $C^{1}(\Sigma)$ ) of energy and volume as defined above. In addition, however, we must bound the higher order terms of these functionals by certain integrals, to obtain information in $C^{1}(\Sigma)$ from spectral theory in $H^{1}(\Sigma)$. The bounds obtained are based on a Taylor expansion. The point of the next few lemmas is to give the remainder of various expansions in a form that we can easily bound for functions which are small in $C^{1}(\Sigma)$. 
Lemma 2.1. Let $\mathcal{S}$ be a surface in $\mathbb{R}^{3}$ parameterized by $p(u, v),(u, v)$ in some parameter domain $D \subseteq \mathbb{R}^{2}$. For $\varphi$ in $C^{1}(\Sigma)$, define $\tilde{\varphi}(u, v)$ to be $\varphi(p(u, v))$. Suppose that $F: C^{1}(\Sigma) \rightarrow \mathbb{R}$ is defined by

$$
F(\varphi)=\iint_{D} f(u, v, \tilde{\varphi}(u, v), \nabla \tilde{\varphi}) d u d v
$$

for some function $f(u, v, w, \vec{\alpha})$ which is three times differentiable in $w$ and $\vec{\alpha}$, with bounded third derivatives. Then we may expand $F(\varphi)$ as

$$
F(\varphi)=F(o)+\iint_{\mathcal{S}} L(\varphi, \nabla \varphi) d \Sigma+\iint_{\mathcal{S}} Q(\varphi, \nabla \varphi) d \Sigma+\eta
$$

where $L$ is a linear operator, $Q$ is a quadratic form, (the coefficients of $L$ and $Q$ depend continuously on $p \in \mathcal{S}$ ), and $\eta$ may be bounded by

$$
|\eta| \leq C \iint_{\mathcal{S}}(|\varphi|+|\nabla \varphi|)^{3} d \Sigma
$$

for some constant $C$. Here $\nabla \varphi$ is the gradient on the surface.

Proof. Holding $u$ and $v$ fixed, we may expand $f(u, v, \tilde{\varphi}, \nabla \tilde{\varphi})$ in the last two variables as

$$
f(u, v, \tilde{\varphi}, \nabla \tilde{\varphi})=f(u, v, 0, \overrightarrow{0})+\tilde{L}(\tilde{\varphi}, \nabla \tilde{\varphi})+\tilde{Q}(\tilde{\varphi}, \nabla \tilde{\varphi})+\eta_{1}
$$

where $\tilde{L}$ is linear, $\tilde{Q}$ is quadratic, and the error term $\eta_{1}$ involves third order terms in $\tilde{\varphi}$ and $\nabla \tilde{\varphi}$ with coefficients consisting of third derivatives of $f$. Since the third derivatives are bounded, we may estimate $\left|\eta_{1}\right|$ by $C(|\tilde{\varphi}|+|\nabla \tilde{\varphi}|)^{3}$ for some constant $C$.

We now integrate (2.3) over $D$. The integrals over $D$ may be converted to integrals over $\mathcal{S}$ by introducing a factor of $\left\|p_{u} \times p_{v}\right\|$. To relate $\nabla \tilde{\varphi}$ and $\nabla \varphi$, note that for a given $(u, v), \nabla \varphi(p(u, v))$ may be obtained by applying a non-singular linear transformation to $\nabla \tilde{\varphi}(u, v)$ (see [12], volume 4).

We will need a lemma for curves which is analogous to Lemma 2.1.

Lemma 2.2. Suppose that $G$ is a smooth curve parameterized by $\gamma(s), s \in$ $[0,1]$. For $\varphi$ defined on $G$, let $T(\varphi)$ be defined as $\int_{0}^{1} f(s, \varphi(\gamma(s))) d s$, where $f$ has continuous third derivatives. Then

$$
T(\varphi)=T(o)+\int_{G} L(\varphi) d \sigma+\int_{G} Q(\varphi) d \sigma+\eta
$$

where $L$ is linear in $\varphi, Q$ is quadratic, and $\eta$ satisfies the bound

$$
|\eta| \leq C \int_{G}|\varphi|^{3} d \sigma
$$

Proof. This is essentially the same as Lemma 2.1 and is omitted. 
Lemma 2.3. Suppose that $\Sigma$ is a smooth, orientable surface with constant mean curvature $H$ with respect to the normal $\vec{N}$, that the contact angle between $\Sigma$ and $\Lambda$ is constantly $\gamma=\arccos (c) \in(0, \pi)$, and that $\vec{x}: \Sigma \times$ $[-\epsilon, \epsilon] \rightarrow \mathbb{R}^{3}$ is a curvilinear coordinate system satisfying $\vec{x}(p, 0)=p$ and $\vec{x}(p, w) \in \Lambda$ for $p \in \partial \Sigma$ as described above. Then for $|\varphi|$ and $|\nabla \varphi|$ sufficiently (uniformly) small,

$$
\mathcal{E}(\varphi)=\mathcal{E}(o)-2 \iint_{\Sigma} H \varphi d \Sigma+\iint_{\Sigma} Q_{1}(\varphi, \nabla \varphi) d \Sigma+\oint_{\partial \Sigma} Q_{2}(\varphi)+\eta_{1}
$$

where $Q_{1}$ and $Q_{2}$ are quadratic forms and $\eta_{1}$ satisfies the bound

$$
\left|\eta_{1}\right| \leq C\left(\iint_{\Sigma}(|\varphi|+|\nabla \varphi|)^{3} d \Sigma+\oint_{\partial \Sigma}|\varphi|^{3} d \sigma\right) .
$$

Proof. The first order term is essentially derived in [11]: a boundary term will disappear since the contact angle condition is assumed to be met. (The assumption that $\vec{x}_{w} \cdot \vec{N}=1$ is used.) We wish to apply Lemma 2.1 (to $A(\varphi)$ ) and Lemma 2.2 (to $W(\varphi)$ ) to bound the remainder term as above. The first problem is that $\Sigma$ might not be parameterized by a single domain $D$. However, we may certainly cut $\Sigma$ into pieces $\Sigma_{i}$ which are disjoint except for their boundaries, each of which may be parameterized by a domain $D_{i}$. We then sum over $i$, so that this is not a real difficulty.

We next must verify that $A(\varphi)$ may be written in the form of Equation (2.2) on each $D_{i}$. For ease of notation, we shall deal with a single $D_{i}$, and will simply call it $D$, and its image $\Sigma$. Also, for ease of notation, let $\vec{z}(u, v, w)=\vec{x}(p(u, v), w)$. Then the surface $\vec{x}(p, \varphi(p))$ may be written as $\vec{z}(u, v, \tilde{\varphi}(u, v)),(u, v) \in D$. Its area will be

$$
\begin{aligned}
\iint_{D} \|\left[\vec{z}_{u}(u, v, \tilde{\varphi}(u, v))+\vec{z}_{w}(u, v, \tilde{\varphi}(u, v)) \tilde{\varphi}_{u}\right] \\
\quad \times\left[\vec{z}_{v}(u, v, \tilde{\varphi}(u, v))+\vec{z}_{w}(u, v, \tilde{\varphi}(u, v)) \tilde{\varphi}_{v}\right] \| d u d v
\end{aligned}
$$

The differentiability assumptions on $\vec{z}(u, v, w)$ will cause the integrand of (2.7) to be a differentiable function of the variables $u, v, \varphi, \varphi_{u}$ and $\varphi_{v}$ as long as the length of the vector

$$
\frac{\partial}{\partial u} \vec{z}(u, v, \varphi(u, v)) \times \frac{\partial}{\partial v} \vec{z}(u, v, \varphi(u, v))
$$

is non-zero. However, $\vec{z}(u, v, 0)$ simply parameterizes $\Sigma$. For this to be a good parameterization, $\vec{z}_{u}(u, v, 0) \times \vec{z}_{v}(u, v, 0) \neq \overrightarrow{0}$. This implies that the integrand of (2.7) is differentiable for $\varphi$ and $\nabla \varphi$ sufficiently small.

We must also show that $W(\varphi)$ (the area of the region on $\Lambda$ wetted by $\vec{x}(p, \varphi(p)))$ may be expanded as in Equation (2.4). If we perturb $\Sigma$ to the surface $\vec{x}(p, \varphi(p))$, then the part of the wetted region on $\Lambda$ within $\epsilon$ of the 
original surface may be parameterized as $\vec{x}(p, w), p \in \partial \Sigma,-\epsilon<w<\varphi(p)$. We may neglect any part of $\Lambda$ farther than $\epsilon$ from $\Sigma$, since any point on $\Lambda$ farther than $\epsilon$ from $\Sigma$ will be wetted by either both the original drop and the perturbed drop or by neither. Thus, we must show that the area of the surface parameterized by $\vec{x}(p, w), p \in \partial \Sigma,-\epsilon<w<\varphi(p)$ may be written in a form to which Lemma 2.2 applies.

Since $\Sigma$ intersects $\Lambda$ transversely, $\partial \Sigma$ consists of smooth curves. Let $p(s)$, $s \in[0,1]$ be a parameterization of one such curve. Then one piece of the wetted surface is $\vec{x}(p(s), w), s \in[0,1], w \in[-\epsilon, \varphi(p(s))]$. Its area is

$$
\int_{0}^{1} \int_{0}^{\varphi(p(s))}\left\|\frac{\partial}{\partial s} \vec{x}(p(s), w) \times \frac{\partial}{\partial w} \vec{x}(p(s), w)\right\| d w d s .
$$

For the inner integrand to be a differentiable function of $s$ and $\varphi(p(s))$, we must have the length of the vector

$$
\frac{\partial}{\partial s} \vec{x}(p(s), w) \times \frac{\partial}{\partial w} \vec{x}(p(s), w)
$$

bounded from zero. The first vector is a non-trivial linear combination of $\vec{x}_{u}$ and $\vec{x}_{v}$, and, by the assumption that $\vec{x}(u, v, w)$ is smooth and invertible, cannot be parallel to $\vec{x}_{w}$. Therefore the integrand in Equation (2.8) is a differentiable function of $s$ and $w$. From this, we have that

$$
\int_{0}^{\varphi(p(s))}\left\|\frac{\partial}{\partial s} \vec{x}(p(s), w) \times \frac{\partial}{\partial w} \vec{x}(p(s), w)\right\| d w
$$

satisfies the assumption of Lemma 2.2. Summing over the smooth pieces of $\partial \Sigma$ concludes the proof.

Lemma 2.4. $V(\varphi)$ may be expanded as

$$
V(\varphi)=V(o)+\iint_{\Sigma} \varphi d \Sigma+\iint_{\Sigma} Q_{3}(\varphi, \nabla \varphi) d \Sigma+\eta_{2}
$$

where $\eta_{2}$ satisfies the bound

$$
\left|\eta_{2}\right| \leq C\left(\iint_{\Sigma}(|\varphi|+|\nabla \varphi|)^{3} d \Sigma+\oint_{\partial \Sigma}|\varphi|^{3} d \sigma\right),
$$

and $Q_{3}$ is a quadratic form.

Proof. If $\Omega$ is the region occupied by the liquid, its volume is

$$
V=\frac{1}{3} \iint_{\partial \Omega} \vec{x} \cdot \vec{N} d \Sigma
$$

by the divergence theorem. The part of (2.10) over the perturbed free surface $\vec{x}(p, \varphi(p)), p \in \Sigma$ may be handled as in Lemma 2.1. The part of (2.10) which is contained in the fixed surface $\Lambda$ is handled as in Lemma 2.3. As this is routine, the details are omitted. The exact form of the first order term is well known. 
Lemma 2.5. Suppose that $\Sigma$ is a capillary surface with contact angle $\gamma \in$ $(0, \pi)$. If $\varphi \in C^{1}(\bar{\Sigma})$ is such that $V(\varphi)=V(o)$, then

$$
\mathcal{E}(\varphi)=\mathcal{E}(o)+\frac{1}{2} \mathcal{M}(\varphi, \varphi)+\eta
$$

where $\eta$ is bounded as in (2.6). $\mathcal{M}(\varphi, \varphi)$ is a quadratic form whose associated bilinear form is given by

$$
\mathcal{M}(\varphi, \psi)=\iint_{\Sigma} \nabla \varphi \cdot \nabla \psi-|S|^{2} \varphi \psi d \Sigma+\oint_{\partial \Sigma} \rho \varphi \psi d \sigma
$$

where $\nabla$ is the gradient on $\Sigma$ and $|S|^{2}$ is the square of the norm of the second fundamental form of $\Sigma$. (In terms of $H$ and the Gaussian curvature $K,|S|^{2}$ may be written as $2\left(2 H^{2}-K\right)$, and in terms of the principal curvatures, $|S|^{2}$ may be written as $k_{1}^{2}+k_{2}^{2}$.) The coefficient $\rho$ is given by

$$
\rho=\kappa_{\Sigma} \cot \gamma-\kappa_{\Lambda} \csc \gamma
$$

where $\kappa_{\Sigma}$ is the curvature of the curve $\Sigma \cap \Pi$ and $\kappa_{\Lambda}$ is the curvature of $\Lambda \cap \Pi$, if $\Pi$ is a plane normal to the contact curve $\partial \Sigma$.

Proof. Since $V(\varphi)$ is assumed to equal $V(o)$, we may apply Lemma 2.4 to find that

$$
\iint_{\Sigma} \varphi d \Sigma=-\iint_{\Sigma} Q_{3}(\varphi, \nabla \varphi) d \Sigma-\eta_{2}
$$

Substituting this into (2.5), we obtain (2.11), where $\mathcal{M}(\varphi, \varphi)$ must equal the integral of a quadratic form in $\varphi$ and $\nabla \varphi$ over $\Sigma$ plus the integral of a quadratic form in $\varphi$ over $\partial \Sigma$.

Having this expansion, we may apply the derivation of [11] or [20] to determine $\mathcal{M}$ explicitly. Indeed, both of these references consider a continuum of constant volume surfaces, parameterized by $t$, so that the unperturbed surface occurs for $t=0$. The quadratic form they obtain is then the second derivative of energy at $o$. Since we have seen that $\mathcal{E}$ may be written as in (2.11), $\eta$ will disappear in taking the second derivative. Thus, $\mathcal{M}$ will be the same as the standard quadratic form found in [11] and [20], and may be written as (2.12).

A quadratic form $M(u, u)$ defined on a Banach space is said to be strongly positive if there is a constant $c>0$ so that $M(u, u) \geq c\|u\|^{2}$ for all $u$ in the space. We will next see that strong positivity of $\mathcal{M}$ on a certain subspace of $H^{1}(\Sigma)$ implies that $\Sigma$ is a local energy minimum under the volume constraint.

Theorem 2.1. Suppose that $\Sigma$ is a capillary surface, with contact angle $\gamma \in$ $(0, \pi)$. If $\mathcal{M}(\varphi, \varphi)$ is strongly positive on the subspace of $H^{1}(\Sigma)$ consisting of all $\varphi$ for which $\iint_{\Sigma} \varphi d \Sigma=0$, then o is a strict local minimum $\left(\right.$ in $\left.C^{1}(\Sigma)\right)$ for $\mathcal{E}$, subject to the volume constraint $V(\varphi)=V(o)$, hence $\Sigma$ is a constrained 
local energy minimum (comparing with nearby surfaces that are of the form $\vec{x}(p, \varphi(p)))$.

Proof. Suppose that $\varphi$ satisfies $V(\varphi)=V(o)$. We cannot apply the assumption of strong positivity of $\mathcal{M}$ directly to this $\varphi$, since there is no reason to expect that $\iint_{\Sigma} \varphi d \Sigma=0$. However, we may write $\varphi$ as $\varphi^{*}+\alpha$, where $\iint_{\Sigma} \varphi^{*} d \Sigma=0$ and $\alpha$ is the constant

$$
\alpha=\frac{\iint_{\Sigma} \varphi d \Sigma}{|\Sigma|}=-\frac{1}{|\Sigma|} \iint_{\Sigma} Q_{3} d \Sigma-\frac{\eta_{2}}{|\Sigma|},
$$

where the second equality comes from Lemma 2.4.

We need to relate the $H^{1}$ norm of $\varphi$ with the $H^{1}$ norm of $\varphi^{*}$. There holds

$$
\iint_{\Sigma} \varphi^{2} d \Sigma=\iint_{\Sigma}\left(\varphi^{*}\right)^{2} d \Sigma+\alpha^{2}|\Sigma|
$$

and therefore (since $\left.\nabla \varphi=\nabla \varphi^{*}\right)$

$$
\|\varphi\|_{1}^{2}=\left\|\varphi^{*}\right\|_{1}^{2}+\alpha^{2}|\Sigma|
$$

where $\|\varphi\|_{1}=\sqrt{\iint_{\Sigma} \varphi^{2}+\iint_{\Sigma}|\nabla \varphi|^{2}}$ is the norm of $\varphi$ in the space $H^{1}(\Sigma)$.

From (2.13) one sees that, for a non-trivial $\varphi$ with $\|\varphi\|_{1}$ small enough, $|\alpha|<C\|\varphi\|_{1}^{2}$ holds for some constant $C$. Thus, if $\|\varphi\|_{1}$ is sufficiently small,

$$
\alpha^{2}|\Sigma|<\frac{1}{2}\|\varphi\|_{1}^{2}
$$

and therefore for $\|\varphi\|_{1}$ sufficiently small,

$$
\frac{1}{2}\|\varphi\|_{1}^{2}<\left\|\varphi^{*}\right\|_{1}^{2}<\|\varphi\|_{1}^{2}
$$

holds as long as $\varphi$ is non-trivial.

For a non-trivial function $\varphi$ satisfying $V(\varphi)=V(o)$, we may use Lemma 2.5 to conclude that

$$
\begin{aligned}
\mathcal{E}(\varphi) & =\mathcal{E}(o)+\frac{1}{2} \mathcal{M}\left(\varphi^{*}+\alpha, \varphi^{*}+\alpha\right)+\eta(\varphi) \\
& =\mathcal{E}(o)+\frac{1}{2} \mathcal{M}\left(\varphi^{*}, \varphi^{*}\right)+\mathcal{M}\left(\varphi^{*}, \alpha\right)+\frac{1}{2} \mathcal{M}(\alpha, \alpha)+\eta(\varphi) \\
& >\mathcal{E}(o)+\frac{c}{2}\left\|\varphi^{*}\right\|_{1}^{2}+\mathcal{M}\left(\varphi^{*}, \alpha\right)+\frac{1}{2} \mathcal{M}(\alpha, \alpha)+\eta(\varphi),
\end{aligned}
$$

where the constant $c>0$ is from the definition of $\mathcal{M}$ being strongly positive. (For the rest of the proof we will assume that $\varphi$ is not trivial.) We will show that the term containing $\left\|\varphi^{*}\right\|_{1}^{2}$ dominates the terms involving $\mathcal{M}$ on the right hand side of (2.15). In other words, we will show that there is a $\beta\left(\varphi^{*}\right)$ so that

$$
\left|\mathcal{M}\left(\varphi^{*}, \alpha\right)+\frac{1}{2} \mathcal{M}(\alpha, \alpha)\right|<\beta\left(\varphi^{*}\right)\left\|\varphi^{*}\right\|_{1}^{2}
$$


with $\beta\left(\varphi^{*}\right)$ tending to 0 as $\left\|\varphi^{*}\right\|_{1}$ goes to zero.

For the term $\mathcal{M}\left(\varphi^{*}, \alpha\right)$, we have

$$
\mathcal{M}\left(\varphi^{*}, \alpha\right)=\alpha\left(\iint_{\Sigma}-|S|^{2} \varphi^{*} d \Sigma+\oint_{\partial \Sigma} \rho \varphi^{*} d \sigma\right) .
$$

Since $|\alpha|<C\|\varphi\|_{1}^{2}<2 C\left\|\varphi^{*}\right\|_{1}^{2}$, to obtain the bound for $\mathcal{M}\left(\varphi^{*}, \alpha\right)$ necessary for (2.16) we will show that $\iint_{\Sigma}|S|^{2} \varphi^{*} d \Sigma$ and $\oint_{\partial \Sigma} \rho \varphi^{*} d \sigma$ are bounded by a constant times $\left\|\varphi^{*}\right\|_{1}$. The argument for the first integral is obvious, and the argument for the second integral uses the fact that the trace map from $H^{1}(\Sigma)$ to $H^{1 / 2}(\partial \Sigma)$ is continuous. The term $\mathcal{M}(\alpha, \alpha)$ is clearly bounded by a constant times $\left\|\varphi^{*}\right\|_{1}^{4}$, so that we obtain that

$$
\begin{aligned}
\mathcal{E}(\varphi) & >\mathcal{E}(o)+\left\|\varphi^{*}\right\|_{1}^{2}\left(\frac{c}{2}+\beta\left(\varphi^{*}\right)\right)+\eta(\varphi) \\
& >\mathcal{E}(o)+c_{1}\left\|\varphi^{*}\right\|_{1}^{2}+\eta(\varphi)
\end{aligned}
$$

for $\|\varphi\|_{1}$ sufficiently small, and for a constant $c_{1}>0$ less than $\frac{c}{2}$.

In general, $\eta(\varphi)$ will not be small even if $\varphi$ is small in $H^{1}(\Sigma)$. However, if we require that $\varphi$ be small in $C^{1}(\Sigma)$, then we can dominate $\eta(\varphi)$ by $\|\varphi\|_{1}^{2}$. Indeed, if we take an $\epsilon>0$, then for a $\varphi$ with $|\varphi|<\epsilon$ and $|\nabla \varphi|<\epsilon$ we have

$$
|\eta(\varphi)|<C \epsilon\left(\|\varphi\|_{1}^{2}+\oint_{\partial \Sigma} \varphi^{2} d \sigma\right) .
$$

Since the trace map is continuous from $H^{1}(\Sigma)$ to $H^{1 / 2}(\partial \Sigma)$, we conclude from (2.18) that there is a $C>0$ so that if $|\varphi|<\epsilon$ and $|\nabla \varphi|<\epsilon$ in $\Sigma$, then

$$
|\eta(\varphi)|<C \epsilon\|\varphi\|_{1}^{2}<C^{\prime} \epsilon\left\|\varphi^{*}\right\|_{1}^{2} .
$$

From (2.19) and (2.17), we conclude that if the $C^{1}(\Sigma)$ norm of $\varphi$ is sufficiently small, and if $V(\varphi)=V(o)$, then we have

$$
\mathcal{E}(\varphi)>\mathcal{E}(o)+c_{2}\left\|\varphi^{*}\right\|_{1}^{2}
$$

holding for non-trivial $\varphi$. (We have again taken a smaller constant times $\left\|\varphi^{*}\right\|_{1}^{2}$ to enable us to drop terms which are dominated by $\left\|\varphi^{*}\right\|_{1}^{2}$.) To conclude the proof, we must show that if $\varphi$ is not identically zero, then $\varphi^{*}$ is not identically zero. This follows from (2.14). Therefore, for any non-trivial $\varphi$ with $V(\varphi)=V(o)$ and of small enough $C^{1}(\Sigma)$ norm, we have

$$
\mathcal{E}(\varphi)>\mathcal{E}(o)
$$

as desired.

We are also able to show instability in certain cases.

Lemma 2.6. If $\Sigma$ is capillary, and if there exists a function $\varphi$ in $H^{1}(\Sigma)$ for which $\iint_{\Sigma} \varphi d \Sigma=0$ and $\mathcal{M}(\varphi, \varphi)<0$, then o is not a constrained minimum for energy in any neighborhood of the origin. In other words, there will be functions, arbitrarily close to zero in the $C^{1}(\Sigma)$ norm, satisfying the volume 
constraint, and with smaller energy than $\mathcal{E}(o)$. This implies that there are surfaces, arbitrarily close to $\Sigma$, with smaller energy than $\Sigma$.

Proof. Inspired by the proof of Lemma 2.4 of [2], we consider the function

$$
f(t, u)=V(t \varphi+u)
$$

and the set $\{(t, u): f(t, u)=V(o)\}$ in the $t u$ plane. From Lemma 2.4, $f_{t}(0,0)=\iint_{\Sigma} \varphi d \Sigma=0$ and $f_{u}(0,0)=\iint_{\Sigma} 1 d \Sigma \neq 0$. Using the Implicit Function Theorem, we may solve $f(t, u)=V(o)$ for $u$ as a function of $t$ in some neighborhood of the origin. For this function, we must have $u^{\prime}(0)=0$ and

$$
V(t \varphi+u(t))=V(o)
$$

for all $t$ sufficiently small. Then, using Lemma 2.5,

$$
\begin{aligned}
\mathcal{E}(t \varphi+u(t))= & \mathcal{E}(o) \\
+ & \frac{1}{2}\left(\iint_{\Sigma} t^{2}|\nabla \varphi|^{2}-|S|^{2}\left(t^{2} \varphi^{2}+2 t u(t) \varphi+u^{2}(t)\right) d \Sigma\right. \\
& \left.+\oint_{\partial \Sigma} \rho\left(t^{2} \varphi^{2}+2 t u(t) \varphi+u^{2}(t)\right) d \sigma\right)+\eta,
\end{aligned}
$$

where $|\eta| \leq C t^{3}$. Thus,

$$
\mathcal{E}(t \varphi+u(t))=\mathcal{E}(o)+t^{2} \mathcal{M}(\varphi, \varphi)+O\left(t^{3}\right)
$$

and for all $t \neq 0$ sufficiently small, $\mathcal{E}(t \varphi+u(t))<\mathcal{E}(o)$. Therefore $o$ is not a constrained local minimum for energy.

The remainder of this section is devoted to finding conditions under which the hypotheses of Theorem 2.1 will be satisfied. The bilinear form $\mathcal{M}(\varphi, \psi)$ is bounded on the Hilbert space $H^{1}(\Sigma)$. Therefore (see [9]) there is a unique bounded linear operator $A: H^{1}(\Sigma) \rightarrow H^{1}(\Sigma)$ which satisfies

$$
\langle\varphi, A \psi\rangle=\mathcal{M}(\varphi, \psi),
$$

where $\langle\varphi, \psi\rangle=\iint_{\Sigma} \varphi \psi+\iint_{\Sigma} \nabla \varphi \cdot \nabla \psi$ is the inner product of $H^{1}(\Sigma)$. We introduce the operator $A$ since the sign of the quadratic term in (2.6) and the spectrum of the operator $A$ will be shown to be related.

Lemma 2.7. The operator A determined by equation (2.20) may be written as $I+T$, where $I$ is the identity and $T$ is a compact operator.

Proof. If $T=A-I$, then $T$ satisfies

$$
\langle\varphi, T \psi\rangle=\iint_{\Sigma}\left(-|S|^{2}-1\right) \varphi \psi d \Sigma+\oint_{\partial \Sigma} \rho \varphi \psi d \sigma .
$$

The result then follows quickly from the fact the natural inclusion of a Sobolev space into a lower order Sobolev space is compact. 
We may apply the well-known spectral theory of compact operators (see, e.g., [9]) to conclude that the spectrum of $A$ consists of countably many eigenvalues, which we will call $\mu_{0}, \mu_{1}, \ldots$, whose corresponding eigenspaces have finite dimension, plus possibly the point 1 . The only possible accumulation point of the eigenvalues of $A$ is 1 . The operator $A$ is a bit unusual, and a major point of this paper is that we may learn about the spectrum of $A$ by dealing with a related eigenvalue problem for a differential operator. Let $\mathcal{L}: H^{1}(\Sigma) \rightarrow H^{-1}(\Sigma)$ be given by

$$
\mathcal{L}(\psi)=-\Delta \psi-|S|^{2} \psi
$$

where $\Delta$ is the Laplace-Beltrami operator on $\Sigma$. We consider the eigenvalue problem given by

$$
\mathcal{L}(\psi)=\lambda \psi
$$

on $\Sigma$, with

$$
\mathbf{b}(\psi) \equiv \psi_{1}+\rho \psi=0
$$

on $\partial \Sigma$, where $\psi_{1}$ is the outward normal derivative of $\psi$. The reason that $\mathcal{L}$ is related to $A$ is that applying Green's formula ([3]) to (2.20) yields

$$
\langle\varphi, A \psi\rangle=\iint_{\Sigma} \varphi \mathcal{L}(\psi) d \Sigma+\oint_{\partial \Sigma} \varphi \mathbf{b}(\psi) d \sigma .
$$

The point of working with $(2.22),(2.23)$ is that eigenvalue problems for partial differential equations are well studied (see e.g., [4]). The spectrum for this problem is discrete, with $\lambda_{0}<\lambda_{1} \leq \lambda_{2} \leq \cdots, \lambda_{n} \rightarrow \infty$ as $n \rightarrow \infty$, and the eigenfunctions $\left\{\varphi_{i}\right\}$ of $(2.22),(2.23)$ may be normalized to form an orthonormal basis of $L^{2}(\Sigma)$. As in [17], we are interested in the relationship between the negative eigenvalues of the problem (2.22), (2.23) and the negative eigenvalues of $A$.

Lemma 2.8. The eigenvalue problem (2.22), (2.23) and the operator $A$ have the same number of negative and non-positive eigenvalues (counting multiplicity).

Proof. The proof is the same as Lemma 2.5 of [19]. 
Because of the relationship between the spectrum of the operator $A$ (i.e., $\left.\mu_{0}, \mu_{1}, \ldots\right)$ and that of the eigenvalue problem (2.22), (2.23) (i.e., $\lambda_{0}, \lambda_{1}$, ...), we may attempt to determine if a capillary surface is a strict local minimum for energy by studying the latter problem.

Theorem 2.2. Suppose that $\Sigma$ is a (zero gravity) capillary surface (i.e., that the mean curvature of $\Sigma$ is constant, and that the contact angle with a given fixed surface $\Lambda$ is constant). Suppose that the contact angle $\gamma$ is strictly between 0 and $\pi$. Let $\lambda_{0}<\lambda_{1} \leq \lambda_{2} \leq \cdots$ be the spectrum of the eigenvalue problem (2.22), (2.23) on $\Sigma$. There are several cases.

1) If $0<\lambda_{0}$ then $o$ is a constrained strict local minimum for energy in $C^{1}(\Sigma)$, so that $\Sigma$ has strictly less energy than nearby surfaces which are graphs of smooth functions in curvilinear coordinates.

2) If $\lambda_{1}<0$ then $o$ is a saddle point for energy and $\Sigma$ is not a local minimum for energy.

3) If $\lambda_{0}<0<\lambda_{1}$, let $\zeta$ solve $A \zeta=1$. (This equation has a solution, since $A$ is invertible.) Then

a) if $\iint_{\Sigma} \zeta<0$ then o is a constrained strict local minimum for energy in $C^{1}(\Sigma)$ and $\Sigma$ is an energy minimum in the same sense as case 1.

b) if $\iint_{\Sigma} \zeta>0$ then o is a saddle point for energy and $\Sigma$ is not a local minimum for energy.

4) If $0=\lambda_{0}$ and if $\iint_{\Sigma} \varphi_{0} \neq 0$ (where $\varphi_{0}$ is the eigenfunction (for (2.22), (2.23) corresponding to $\left.\lambda_{0}\right)$ ) then o is a strict local minimum for energy, and $\Sigma$ is an energy minimum as in case 1 .

Proof.

1) It follows from Lemma 2.8 that $\sigma(A)$ (the spectrum of $A$ ) is contained in $(0, \infty)$. Since the only cluster point of $\sigma(A)$ is 1 , this implies that $\sigma(A) \subseteq\left[\mu_{0}, \infty\right) \subseteq(0, \infty)$, where $\mu_{0}>0$ is the smallest eigenvalue of $A$. From this we have that

$$
\mu_{0}\|\varphi\|_{1}^{2} \leq \mathcal{M}(\varphi, \varphi)
$$

for all $\varphi \in H^{1}(\Sigma)$ which is more than enough for Theorem 2.1 to imply the desired result.

2) It is easy to verify that some non-trivial linear combination of $\varphi_{0}$ and $\varphi_{1}$ will satisfy

$$
\iint_{\Sigma} c_{0} \varphi_{0}+c_{1} \varphi_{1} d \Sigma=0
$$


Then

$$
\begin{aligned}
\mathcal{M}\left(c_{0} \varphi_{0}+c_{1} \varphi_{1}, c_{0} \varphi_{0}+c_{1} \varphi_{1}\right)= & \left\langle c_{0} \varphi_{0}+c_{1} \varphi_{1}, A\left(c_{0} \varphi_{0}+c_{1} \varphi_{1}\right)\right\rangle \\
= & \iint_{\Sigma}\left(c_{0} \varphi_{0}+c_{1} \varphi_{1}\right) \mathcal{L}\left(c_{0} \varphi_{0}+c_{1} \varphi_{1}\right) d \Sigma \\
& +\oint_{\partial \Sigma}\left(c_{0} \varphi_{0}+c_{1} \varphi_{1}\right) \mathbf{b}\left(c_{0} \varphi_{0}+c_{1} \varphi_{1}\right) d \sigma \\
= & \lambda_{0} c_{0}^{2}+\lambda_{1} c_{1}^{2}<0
\end{aligned}
$$

and the result follows from Lemma 2.6.

3) a) (Note that $\iint_{\Sigma} \zeta d \Sigma=\langle\zeta, A \zeta\rangle$.) The proof is essentially the same as Theorem 2 of [17], although I will outline it since the notation is different. For more details, please refer to [17].

Take any $\varphi$ which satisfies $\iint_{\Sigma} \varphi d \Sigma=0$. There is an $\alpha$ so that

$$
\varphi=v+\alpha \zeta
$$

where $\left\langle v, \psi_{0}\right\rangle=0$. (To show that such an $\alpha$ exists, it suffices to show that $\left\langle\zeta, \psi_{0}\right\rangle \neq 0$. But if this inner product were zero, it would follow that $\langle\zeta, A \zeta\rangle=\iint_{\Sigma} \zeta d \Sigma>0$, contrary to the assumption of this case.)

Then, as in [17],

$$
0=\iint_{\Sigma} \varphi d \Sigma=\langle\varphi, A \zeta\rangle=\langle v, A \zeta\rangle+\alpha\langle\zeta, A \zeta\rangle
$$

implies that

$$
\langle\varphi, A \varphi\rangle=\langle v, A v\rangle-\alpha^{2}\langle\zeta, A \zeta\rangle \geq\langle v, A v\rangle \geq \mu_{1}\|v\|_{1}^{2} .
$$

One can verify that $\|v\|_{1} \geq k\|\varphi\|_{1}$ for some positive constant $k$. Thus the hypothesis of Theorem 2.1 is met, and $o$ is a strict local minimum.

b) In this case, if

$$
c=\frac{-\iint_{\Sigma} \psi_{0} d \Sigma}{\iint_{\Sigma} \zeta d \Sigma}
$$

then $\iint_{\Sigma} \psi_{0}+c \zeta d \Sigma=0$. We will show that $\mathcal{M}\left(\psi_{0}+c \zeta, \psi_{0}+c \zeta\right)<0$. Note first that $\iint_{\Sigma} \psi_{0}+c \zeta d \Sigma=0$ is the same as

$$
\left\langle\psi_{0}+c \zeta, A \zeta\right\rangle=0
$$

so that

$$
\left\langle\psi_{0}, A \zeta\right\rangle=-c\langle\zeta, A \zeta\rangle=-c \iint_{\Sigma} \zeta d \Sigma
$$


Using (2.25),

$$
\begin{aligned}
\left\langle\psi_{0}+c \zeta, A\left(\psi_{0}+c \zeta\right)\right\rangle & =\left\langle\psi_{0}, A \psi_{0}\right\rangle-c^{2} \iint_{\Sigma} \zeta d \Sigma \\
& =\mu_{0}\left\|\psi_{0}\right\|_{1}^{2}-c^{2} \iint_{\Sigma} \zeta d \Sigma<0
\end{aligned}
$$

which implies that the origin is not a local energy minimum, by Lemma 2.6.

4) Note that if $\mathcal{L}\left(\varphi_{0}\right)=0$ on $\Sigma, \mathbf{b}\left(\varphi_{0}\right)=0$ on $\partial \Sigma$, then $A \varphi_{0}=0$, so that in the special case of $\lambda_{0}=0, \varphi_{0}$ is an eigenfunction for both eigenvalue problems.

We need to verify the hypothesis of Theorem 2.1. Take a function $\varphi \in H^{1}(\Sigma)$ with $\iint_{\Sigma} \varphi d \Sigma=0$. Projecting onto $\varphi_{0}$, we may write $\varphi$ as $c \varphi_{0}+y$, where $\left\langle\varphi_{0}, y\right\rangle=0$. Then

$$
\langle\varphi, A \varphi\rangle=\left\langle c \varphi_{0}+y, A y\right\rangle=\langle y, A y\rangle \geq \mu_{1}\|y\|_{1}^{2},
$$

the last inequality following since $y$ is orthogonal to $\varphi_{0}$. We now have to compare $\|\varphi\|_{1}$ and $\|y\|_{1}$. Applying Lemma 2.3 of [18], there is a positive $\delta$, independent of $\varphi$, so that $\|y\|>\delta\|\varphi\|$. Therefore,

$$
\langle\varphi, A \varphi\rangle \geq \mu_{1} \delta^{2}\|\varphi\|^{2}
$$

for all $\varphi$ with $\iint_{\Sigma} \varphi d \Sigma=0$, so that the result follows from Theorem 2.1.

The following observation may make finding the function $\zeta$ in the previous theorem a bit easier.

Note 2.1. For $\zeta \in H^{1}(\Sigma)$, the following two conditions are equivalent:

1) $A \zeta=1$.

2) $\mathcal{L}(\zeta)=1$ in $\Sigma$ and $\mathbf{b}(\zeta)=0$ on $\partial \Sigma$.

Proof. This follows from Equation (2.24).

\section{Curvilinear coordinates: existence and interpretation.}

The hypotheses of Theorem 2.2 do not refer explicitly to the curvilinear coordinate system $\vec{x}(p, w)$, and I don't anticipate that such a coordinate system will be constructed when this theorem is applied. There are two questions which naturally arise, however. The first is whether such a coordinate system will exist in a given capillary problem, and the second is to understand the stability promised by Theorem 2.2 in the absence of a specific coordinate system.

Starting with the first question, we will outline two situations in which a curvilinear coordinate system of the type required for Theorem 2.2 will exist. 
Proposition 3.1. Suppose that $\Sigma$ and $\Lambda$ are smooth surfaces, that $\Sigma$ is compactly contained in a slightly larger smooth surface, and that the angle of contact $\gamma$ is strictly between 0 and $\pi$. Then a curvilinear coordinate system as described in Section 2 exists.

Proof. Since $\Sigma$ and $\Lambda$ intersect transversely, $\partial \Sigma$ will be a smooth curve. Let $\vec{T}$ be a smooth vector field defined on the closure of $\Sigma$ which is tangent to $\Sigma$, normal to $\partial \Sigma$, of unit length on $\partial \Sigma$, and vanishing outside of a neighborhood of $\partial \Sigma$. For $p \in \partial \Sigma$ and each $w$ sufficiently small, there is a smooth function $t(p, w)$ so that $p+w \vec{N}+t(p, w) \vec{T}$ lies on $\Lambda$. We have that $t(p, 0)=0$ for all $p \in \partial \Sigma$. We may extend $t$ smoothly to all of $\bar{\Sigma} \times(-\epsilon, \epsilon)$ so that $t(p, 0)=0$ for all $p \in \Sigma$.

Define $\vec{x}(p, w)$ by

$$
\vec{x}=p+w \vec{N}(p)+t(p, w) \vec{T}(p),
$$

where $\vec{N}$ is the unit normal to $\Sigma$. It is not hard to show that this is a curvilinear coordinate system of the desired form.

There is a somewhat less explicit way of finding a curvilinear coordinate system which will apply in more generality than Proposition 3.1. It depends on the existence of a vector field $\vec{F}(x, y, z)$ which is transverse to $\Sigma$ and tangent to $\Lambda$. This will apply to some capillary problems in which the fixed surface $\Lambda$ is piecewise smooth.

Lemma 3.1. Suppose that $\Lambda$ is the union of $\Lambda_{1}, \cdots, \Lambda_{m}$, where each $\Lambda_{j}$ is smooth and smoothly extendible (as $\Sigma$ is in Proposition 3.1). Suppose that if $\Lambda_{i} \cap \Lambda_{j} \neq \emptyset$ then $\Lambda_{i}$ intersects $\Lambda_{j}$ transversely, and if the curve $\Lambda_{i} \cap \Lambda_{j}$ intersects $\bar{\Sigma}$ then that intersection is also transverse. Then there exists a smooth vector field $\vec{F}(x, y, z)$ defined in a neighborhood of $\Sigma$ which is tangent to $\Lambda$ and transverse to $\Sigma$.

Proof. Let $\vec{N}$ and $\vec{N}_{i}$ be normal vector fields to $\Sigma$ and the $\Lambda_{i}$ 's respectively. These extend smoothly into $\mathbb{R}^{3}$. In a neighborhood of $\Lambda_{i} \cap \Lambda_{j}, \vec{N}_{i} \times \vec{N}_{j}$ will be transverse to $\Sigma$. Put such neighborhoods around all intersection curves in $\Lambda$. Looking at the part of each $\Lambda_{i}$ outside of these neighborhoods, it is easy to construct a vector field tangent to $\Lambda_{i}$ and transverse to $\Sigma$ (in fact $\left(\vec{N} \times \vec{N}_{i}\right) \times \vec{N}_{i}$ will work). Finally, away from $\Lambda$, we can take $\vec{N}$ itself as the transverse vector field. These may now be pieced together using a partition of unity. As this is routine, details are omitted.

When such a vector field $\vec{F}$ exists, we may use it to construct a curvilinear coordinate system as needed in the previous section.

Proposition 3.2. Suppose that a smooth vector field $\vec{F}$ exists which is transverse to $\Sigma$ and tangent to $\Lambda$. Then there exists a curvilinear coordinate system as described in Section 2. 
Proof. For each $p \in \Sigma$, define $\vec{x}(p, w)$ to be the function of $w$ which solves the ordinary differential equation

$$
\frac{d}{d w} \vec{x}(p, w)=\vec{F}(\vec{x}(p, w))
$$

with initial condition

$$
\vec{x}(p, 0)=p .
$$

Here we are considering $p$ as a parameter in the ODE. Since solutions to ODE's depend smoothly on their parameters, it is not difficult to verify that this will be a curvilinear coordinate system in a neighborhood of $\Sigma$, as desired.

If we do not have an explicit curvilinear coordinate system, it's natural to wonder what the result of Theorem 2.2 means if $o$ is an energy minimum. Clearly there are surfaces which are arbitrarily close to $\Sigma$ which can't be written as $\vec{x}(p, \varphi(p))$ for any function $\varphi$ defined on $\Sigma$. In other words, there are functions $\vec{\xi}(p)$ defined on $\Sigma$ which are uniformly small, but for which the perturbed surface $p+\vec{\xi}(p)$ can't be written as $\vec{x}(p, \varphi(p))$. However, if we restrict $\nabla \vec{\xi}$ as well, it turns out that we will be able to write the perturbed surface as $\vec{x}(p, \varphi(p))$, where $\varphi$ and $\nabla \varphi$ are uniformly small. Of course, this statement needs some proof.

Lemma 3.2. There exists an $\epsilon>0$ so that if $|\vec{\xi}|<\epsilon$ and $|\nabla \vec{\xi}|<\epsilon$ uniformly on $\Sigma$, then $\vec{N}_{\xi} \cdot \vec{x}_{w} \neq 0$. Here $\vec{N}_{\xi}$ is the normal to the perturbed surface $p+\vec{\xi}(p)$.

Proof. Consider a coordinate patch on $\Sigma, p(u, v)$. This induces the natural coordinate patch on $\Sigma_{\xi}$ given by $p(u, v)+\vec{\xi}(p(u, v))$. The result follows from writing out the normal explicitly.

Lemma 3.3. If $\vec{\xi}$ satisfies the conditions of Lemma 3.2, then $\Sigma_{\xi}$ is locally a smooth graph in curvilinear coordinates. In other words, given any $p^{*} \in \Sigma_{\xi}$, there is a neighborhood of $p^{*}$ in which $\Sigma_{\xi}$ is the set of points $\vec{x}(p, \varphi(p))$ for some differentiable function $\varphi$ defined on $\Sigma$. Moreover, $|\varphi|$ and $|\nabla \varphi|$ may be bounded in terms of $|\vec{\xi}|$ and $|\nabla \vec{\xi}|$, with the first two quantities going to zero as the latter two quantities go to zero.

Proof. If we have a smooth surface in the region of curvilinear coordinates given by $\vec{s}(a, b)$ with $a$ and $b$ as parameters, the condition that the equation $\vec{s}(a, b)=\vec{x}(p(u, v), w)$ determines $w$ implicitly as a differentiable function of $u$ and $v$ is precisely that $\vec{s}_{a}, \vec{s}_{b}$ and $\vec{x}_{w}$ are linearly independent, i.e., that $\vec{x}_{w}$ is not in the tangent space to the surface. (This statement uses the assumption that $\vec{x}(p(u, v), w)$ is a smooth invertible map from $\mathbb{R}^{3}$ to $\mathbb{R}^{3}$.) The linear independence holds if $\vec{x}_{w}$ is not orthogonal to the normal to the surface. The first part of the result now follows from the previous lemma. 
The bound for $|\varphi|$ can be obtained by continuity, since if $\vec{\xi}$ is identically zero, so is $\varphi$. A bound for $|\nabla \varphi|$ may be obtained by writing this gradient out explicitly using the implicit function theorem, although we will omit the details.

The above lemma is not quite what we want, however, since the implicit function theorem is a local result. We need to show that there is a single function $\varphi$ so that $\Sigma_{\xi}$ may be written as $\vec{x}(p, \varphi(p))$, i.e., we need to extend the local statement of Lemma 3.3 to a global statement. To this end, we define a function $n(p)$ on $\Sigma$ to be the number of points $p^{*}$ so that there exists a $w$ with $\vec{x}(p, w)=p^{*}+\vec{\xi}\left(p^{*}\right) . \quad \Sigma_{\xi}$ is a graph in curvilinear coordinates if and only if $n(p)$ is identically 1 on $\Sigma$.

Lemma 3.4. If $\vec{\xi}$ satisfies the hypotheses of Lemma 3.2 then $n(p)$ is continuous on $\Sigma$, and therefore constant on connected components of $\Sigma$.

Proof. Suppose $n(p)=k$, and let $q_{1}^{*}, \cdots, q_{k}^{*}$ be the corresponding $k$ points on $\Sigma$. From Lemma 3.3, there are $k$ neighborhoods $\Omega_{k}$ on $\Sigma_{\xi}$ of the points $q_{j}^{*}+\vec{\xi}\left(q_{j}^{*}\right)$ in which $\Sigma_{\xi}$ is a graph over a neighborhood of $p$. Take the intersection of the preimages of these neighborhoods to see that $n(p)$ is at least $k$ in a neighborhood of $p$. Thus $n(p)$ is lower-semicontinuous on $\Sigma$. Now suppose (by way of contradiction) that there is a sequence $p_{i}$ of points of $\Sigma$ approaching $p$ so that $n\left(p_{i}\right)>k$ for all $i$. For each point $p_{i}$ there is a point $p_{i}^{*}$ in none of the neighborhoods $\Omega_{k}$ for which

$$
p_{i}^{*}+\vec{\xi}\left(p_{i}^{*}\right)=\vec{x}\left(p_{i}, w_{i}\right)
$$

for some $w_{i}$. By a compactness argument, we may take subsequences so that $p_{i}^{*} \rightarrow p^{*} \in \bar{\Sigma}$ and $w_{i} \rightarrow w \in[-\epsilon, \epsilon]$. Taking the limit in (3.1) we find that $p^{*}+\vec{\xi}\left(p^{*}\right)=\vec{x}(p, w)$. This is a contradiction, since $p^{*}$ cannot equal any of the points $q_{1}^{*}, \cdots, q_{k}^{*}$. Thus $n(p)$ is continuous.

Theorem 3.1. There exists an $\epsilon>0$ so that for any $\vec{\xi}$ defined on $\Sigma$ with $|\vec{\xi}|<\epsilon$ and $|\nabla \vec{\xi}|<\epsilon$, the surface $\Sigma_{\xi}$ may be written as the graph of a differentiable function in curvilinear coordinates. (By "the surface $\Sigma_{\xi}$ ", we mean the set of points $p+\vec{\xi}(p)$, where $p$ ranges over all of $\Sigma$.)

Proof. This will follow if it can be shown that for $\epsilon$ sufficiently small, $|\vec{\xi}|<\epsilon$ and $|\nabla \vec{\xi}|<\epsilon$ imply that $n(p)=1$ everywhere on $\Sigma$. If this is not the case, then there is a sequence $\vec{\xi}_{k}$ with $\left|\vec{\xi}_{k}\right| \rightarrow 0,\left|\nabla \vec{\xi}_{k}\right| \rightarrow 0$, but $n_{k}(p)$ is not identically 1 for all $k$. An area argument shows that this cannot happen. Since $\vec{\xi}_{k}$ and $\nabla \vec{\xi}_{k}$ go uniformly to zero, the area of $\Sigma_{\xi_{k}}$ must approach the area of $\Sigma$, since the integrand in the area integrals will converge uniformly. On the other hand, if $n_{k}(p)$ is not identically 1 , the areas of the $\Sigma_{\xi_{k}}$ 's cannot approach any number smaller that the area of $\Sigma$ plus the area of the smallest connected component of $\Sigma$. This contradiction proves the theorem. 
To conclude, we will now interpret Theorem 2.2 in light of the above result, thus yielding a result which does not refer to a specific curvilinear coordinate system.

Theorem 3.2. If a capillary surface $\Sigma$ satisfies the criteria in Theorem 2.2 for being a strict local energy minimum in curvilinear coordinates, then $\Sigma$ is a strict local minimum for energy subject to the volume constraint in the following sense. There is an $\epsilon>0$ so that for any $\vec{\xi}$ defined on $\Sigma$ with $|\vec{\xi}|<\epsilon,|\nabla \vec{\xi}|<\epsilon$, and $\Sigma_{\xi}$ surrounding the same volume as $\Sigma$, we must have either that $\Sigma_{\xi}$ has a strictly larger energy than $\Sigma$ or that $\Sigma$ and $\Sigma_{\xi}$ are the same set of points in $\mathbb{R}^{3}$.

Acknowledgment. I am grateful to the referees for a number of helpful and insightful comments.

\section{References}

[1] V.M. Alekseev, V.M. Tikhomirov and S.V. Fomin, Optimal Control, Plenum Publishing Corporation, New York, NY, 1987.

[2] J.L. Barbosa and M. do Carmo, Stability of hypersurfaces with constant mean curvature, Math. Z., 185 (1984), 339-353.

[3] I. Chavel, Eigenvalues in Riemannian Geometry, Academic Press, New York, 1984.

[4] R. Courant and D. Hilbert, Methods of Mathematical Physics, Vol. 1. Interscience Publishers, New York, NY, 1953.

[5] R. Finn, Equilibrium Capillary Surfaces, Springer-Verlag, New York, NY, 1986.

[6] _ Editorial comments on "On stability of a catenoidal liquid bridge", by L. Zhou, Pacific J. Math., 178(1) (1997), 197-198.

[7] D. Gilbarg and N.S. Trudinger, Elliptic Partial Differential Equations of Second Order, Springer-Verlag, New York, N.Y., second edition, 1983.

[8] K. Große-Brauckmann, Stable constant mean curvature surfaces minimize area, Pacific J. Math., 175(2) (1996), 526-534.

[9] G. Helmberg, Introduction to Spectral Theory in Hilbert Space, North Holland, Amsterdam, 1969.

[10] J.H. Maddocks, Stability and folds, Arch. Rat. Mech. Anal., 99 (1987), 301-328.

[11] A. Ros and R. Souam, On stability of capillary surfaces in a ball, Pacific J. Math., 178(2) (1997), 345-361.

[12] M. Spivak, A Comprehensive Introduction to Differential Geometry, Publish or Perish, Inc., Boston, MA, 1975.

[13] J.E. Taylor, Boundary regularity for solutions to various capillary and free boundary problems, Comm. Partial Differential Equations, 2(4) (1977), 323-357.

[14] T.I. Vogel, Stability of a liquid drop trapped between two parallel planes, SIAM J. Appl. Math., 47(3) (1987), 516-525.

[15] Uniqueness for certain surfaces of prescribed mean curvature, Pacific J. Math., 134(1) (1988), 197-207. 
[16] , Stability of a liquid drop trapped between two parallel planes II: General contact angles, SIAM J. Appl. Math., 49(4) (1989), 1009-1028.

$[17]$ , On constrained extrema, Pacific J. Math., 176(2) (1996), 557-561.

$[18]$ , Sufficient conditions for multiply constrained extrema, Pacific J. Math., $\mathbf{1 8 0 ( 2 )}$ (1997), 377-384.

[19] Non-linear stability of a certain capillary surface, Dynam. Contin. Discrete Impuls. Systems, 5(1-4) (1999), 1-16.

[20] H.C. Wente, Existence Theorems for Surfaces of Constant Mean Curvature and Perturbations of a Liquid Globule in Equilibrium, Ph.D. thesis, Harvard University, 1966.

[21] L. Zhou, On stability of a catenoidal liquid bridge, Pacific J. Math., 178(1) (1997), 185-197.

Received June 19, 1998 and revised March 16, 1999.

Department of Mathematics

TExas A\&M University

College Station, TX 77843-3368

E-mail address: tvogel@math.tamu.edu 